\title{
Blocking translationally controlled tumor protein attenuate aggressiveness of fibroblast-like synoviocytes and ameliorates collagen-induced arthritis
}

\author{
Mingyo Kim ${ }^{1}$, Yongho Choe ${ }^{1}$, Heewon Lee ${ }^{2}$, Min-Gyu Jeon ${ }^{3}$, Jin-Ho Park ${ }^{3}$, Hae Sook \\ Noh $^{3}$, Yun-Hong Cheon ${ }^{1}$, Hee Jin Park ${ }^{3}$, Kyunglim Park ${ }^{4}$, Sung Jae Shin ${ }^{4}$, Kyunglim Lee², \\ and Sang-Il Lee ${ }^{3}$ \\ ${ }^{1}$ Gyeongsang National University Hospital \\ ${ }^{2}$ Ewha Womans University College of Pharmacy \\ ${ }^{3}$ Gyeongsang National University College of Medicine \\ ${ }^{4}$ Yonsei University College of Medicine
}

June 10, 2020

\begin{abstract}
Background and Purpose Histamine releasing factor/translationally controlled tumor protein (HRF/TCTP) stimulates cancer progression and allergic responses, but the role of HRF/TCTP remains undefined in rheumatoid arthritis (RA). In this study, we explored the pathogenic significance of HRF/TCTP and evaluated the therapeutic effects of HRF/TCTP blockade in RA. Experimental Approach HRF/TCTP transgenic (TG) and knockdown (KD) mice with collagen-induced arthritis (CIA) were used to determine experimental phenotypes of RA. HRF/TCTP levels were measured in the sera of RA patients and compared to those with osteoarthritis (OA), ankylosing spondylitis, Behçet's disease, and healthy controls. HRF/TCTP expression was also assessed in the synovium and fibroblast-like synoviocytes (FLS) obtained from RA or OA patients. Finally, we assessed the effects of HRF/TCTP and dimerized HRF/TCTP-binding peptide-2 (dTBP2), an HRF/TCTP inhibitor, in RA-FLS and CIA mice. Key Results Our clinical, radiological, histological, and biochemical analyses indicate that inflammatory responses and joint destruction were higher in HRF/TCTP TG mice, and lower in KD mice, compared to that in wild-type littermates. HRF/TCTP levels were higher in the sera, synovial fluid, synovium, and FLS of patients with RA than control groups. Serum levels of HRF/TCTP correlated well with disease activity of RA. Tumor-like aggressiveness of RA-FLS was exacerbated by HRF/TCTP stimulation and ameliorated by dTBP2 treatment. dTBP2 exerted protective and therapeutic effects in CIA mice, and had no detrimental effects in a murine tuberculosis model. Conclusion and Implications Our results indicate that $\mathrm{HRF} / \mathrm{TCTP}$ is a novel biomarker and therapeutic target for the diagnosis and treatment of RA.
\end{abstract}

\section{Background and Purpose}

Histamine releasing factor/translationally controlled tumor protein (HRF/TCTP) stimulates cancer progression and allergic responses, but the role of HRF/TCTP remains undefined in rheumatoid arthritis (RA). In this study, we explored the pathogenic significance of HRF/TCTP and evaluated the therapeutic effects of HRF/TCTP blockade in RA.

Experimental Approach

HRF/TCTP transgenic (TG) and knockdown (KD) mice with collagen-induced arthritis (CIA) were used to determine experimental phenotypes of RA. HRF/TCTP levels were measured in the sera of RA patients and compared to those with osteoarthritis (OA), ankylosing spondylitis, Behçet's disease, and healthy controls. HRF/TCTP expression was also assessed in the synovium and fibroblast-like synoviocytes (FLS) obtained 
from RA or OA patients. Finally, we assessed the effects of HRF/TCTP and dimerized HRF/TCTP-binding peptide-2 (dTBP2), an HRF/TCTP inhibitor, in RA-FLS and CIA mice.

\section{Key Results}

Our clinical, radiological, histological, and biochemical analyses indicate that inflammatory responses and joint destruction were higher in HRF/TCTP TG mice, and lower in KD mice, compared to that in wild-type littermates. HRF/TCTP levels were higher in the sera, synovial fluid, synovium, and FLS of patients with RA than control groups. Serum levels of HRF/TCTP correlated well with disease activity of RA. Tumorlike aggressiveness of RA-FLS was exacerbated by HRF/TCTP stimulation and ameliorated by dTBP2 treatment. dTBP2 exerted protective and therapeutic effects in CIA mice, and had no detrimental effects in a murine tuberculosis model.

\section{Conclusion and Implications}

Our results indicate that $\mathrm{HRF} / \mathrm{TCTP}$ is a novel biomarker and therapeutic target for the diagnosis and treatment of RA.

Keywords : Rheumatoid arthritis, Fibroblast-like synoviocytes, Histamine-releasing factor, Translationally controlled tumor protein

\section{Introduction}

Rheumatoid arthritis (RA) is a chronic inflammatory and systemic autoimmune disease affecting $1 \%$ of the general population (Firestein, 2003). RA is characterized by destructive joint inflammation and systemic comorbidities, including cardiovascular complications, psychological impairment, and risk of malignancy (Blum \& Adawi, 2019; Cheon et al., 2018; De Cock \& Hyrich, 2018). Our understanding of RA pathogenesis has improved, and therapeutic strategies against RA have been revolutionized by the development of targeted drugs such as tumor necrosis factor inhibitors, interleukin -6 (IL-6) blockers, B-cell depletion agents, and inhibitors of T-cell costimulation and Janus kinases (Singh et al., 2016). Nevertheless, an appreciable percentage of patients experience refractory disease and interrupt treatment due to risk of infection and malignancy (Favalli et al., 2016; Rutherford, Subesinghe, Hyrich, \& Galloway, 2018). Meanwhile, autoantibodies may be present long before the onset of clinical arthritis; thus, recent investigations have focused on preclinical phases of RA (Mankia \& Emery, 2016; van Steenbergen, da Silva, Huizinga, \& van der Helm-van Mil, 2018). Although several strategies are currently being tested, there is still no effective prevention for RA (9). Thus, the investigation of new cytokines or cellular mechanisms that could be potential targets for RA treatment or prevention remains of high importance.

Pannus is a characteristic feature of RA, and is present at the interface between synovium, cartilage, and bone. Therefore, pannus provides a microenvironment that sustains autoimmunity, and supports cellular and cytokine activity crucial for joint destruction (Orr et al., 2017; You, Koh, Leng, Kim, \& Bucala, 2018). Fibroblast-like synoviocytes (FLS), which are major components of hyperplastic pannus, resemble cancer cells in their resistance to apoptosis, increased migratory and invasive properties, and ability to escape contact inhibition. FLS can destroy bone and cartilage even after their removal from the autoimmune milieu (Bottini \& Firestein, 2013; Bucala, Ritchlin, Winchester, \& Cerami, 1991; Lefevre et al., 2009). The connective-tissue origin of FLS may render them intrinsically resistant to current immunosuppressive drugs. Thus, recurrent or refractory RA likely depends on persistent immunopathologic activity of resident RA-FLS within pannus (Pitzalis, Kelly, \& Humby, 2013). Disrupting FLS-specific immunopathology may prevent joint destruction by mechanisms that do not contribute to systemic immunosuppression and increased infection risk (Hah et al., 2010; Lee et al., 2014; Noss \& Brenner, 2008). Accordingly, interfering with RA-FLS proliferation and invasion may contribute to effective and long-term disease control.

Histamine releasing factor (HRF) was initially classified as a tumor protein (translationally controlled tumor protein, TCTP) in the 1980s, but its function remains unclear (Chitpatima, Makrides, Bandyopadhyay, \& Brawerman, 1988; Yenofsky, Cereghini, Krowczynska, \& Brawerman, 1983). HRF/TCTP triggers histamine 
release from basophils; however, HRF/TCTP also functions similarly to proinflammatory cytokines in several inflammatory and allergic diseases, including asthma, atopic dermatitis, and eosinophilic pneumonia (Sampson, Broadbent, \& Bernhisel-Broadbent, 1989; Warner, Pienkowski, Plaut, Norman, \& Lichtenstein, 1986; Yoneda et al., 2004). A previous study demonstrated that HRF/TCTP inhibits p53-mediated apoptosis, indicating that HRF/TCTP plays a distinctive role in tumorigenicity (Rho et al., 2011). This notion is supported by our previous study, showing that HRF/TCTP induces epithelial-mesenchymal transition (EMT) and promotes pulmonary metastasis of melanoma cells (Bae, Kim, Lee, \& Lee, 2015). Therefore, $\mathrm{HRF} / \mathrm{TCTP}$ is a promising target for prostate, breast, and lung cancer therapies (Acunzo, Baylot, So, \& Rocchi, 2014). Because RA is a representative inflammatory disease, and RA-FLS exhibits p53 dysfunction and undergoes a tumor-like EMT process, HRF/TCTP may potentially serve as a novel therapeutic target in the treatment of RA.

An earlier study has shown that HRF/TCTP is present and localized only in the joints of patients with RA, and not in healthy controls. In particular, HRF/TCTP expression was more pronounced in the FLS of severely destroyed regions of the pannus in RA samples (Maslinska et al., 2008). This finding strongly suggests that HRF/TCTP expression contributes to RA pathogenesis; however, no other studies have examined the functions and relevance of HRF/TCTP in RA. Here, we explore the role of HRF/TCTP in RA using HRF/TCTP transgenic (TG) and knockdown (KD) mice with collagen-induced arthritis (CIA). We also assessed the levels of HRF/TCTP in the sera, joint fluid, synovial tissue, and FLS of patients with RA and in the controls, and examined how HRF/TCTP expression correlate with RA disease activity. Additionally, we evaluated the inhibitory effects of HRF/TCTP-binding peptide on RA-FLS phenotype, and assessed the preventive and therapeutic effects of this peptide in mice with CIA.

\section{Materials and Methods}

\section{Patients}

Patients with RA were classified according to ACR/EULAR 2010 Rheumatoid Arthritis Classification Criteria (Aletaha et al., 2010). The disease activity of RA was determined using the disease activity score (DAS28) using clinical and laboratory data (Fransen \& van Riel, 2009). Patients with osteoarthritis, ankylosing spondylitis, and Behçet's disease were classified according to specific disease classification criteria (Altman et al., 1986; "Criteria for diagnosis of Behcet's disease. International Study Group for Behcet's Disease," 1990; van der Linden, Valkenburg, \& Cats, 1984). All patients provided written informed consent. The biospecimens used in this study were provided by Gyeongsang National University Hospital, a member of the Korea Biobank Network. This study was approved by the Institutional Review Board of Gyeongsang National University Hospital (Permit No: GNUH 2014-02-013).

\section{Animals and experimental protocols}

All mice experimental procedures were approved by the Institutional Animal Care and Use Committee of Gyeongsang National University (Permit No: GNU-150907-M0049). HRF/TCTP KD mice, heterozygous for the deleted HRF/TCTP allele, were kindly provided by Hsin-Fang Yang-Yen (Institute of Molecular Biology, Academia Sinica, Taipei, Taiwan). HRF/TCTP TG mice were generated as previously described (M. J. Kim et al., 2008) and backcrossed with C57BL/6 mice. A previously established protocol with slight modifications was used to induce CIA using C57BL/6 strain mice (Woo et al., 2016; Woo et al., 2018). Wildtype (WT), KD, or TG mice (14-18 weeks old) were immunized with $150 \mu \mathrm{g}$ of chicken type II collagen (CII; Chondrex, Redmond, WA) emulsified in an equal volume of $4 \mathrm{mg} / \mathrm{ml}$ complete Freund's adjuvant (CFA; Chondrex). The day of initial immunization was designated as Day 0. Immunization was boosted by an equal volume of emulsion of CII and incomplete Freund's adjuvant (IFA; Chondrex) on Day 21 and by an intraperitoneal (i.p.) injection of lipopolysaccharide (LPS, $5 \mu \mathrm{g} / \mathrm{mouse}$ ) on Day 28. The clinical arthritis scores, ankle thickness, and micro-computed tomography (micro-CT) imaging were evaluated as described in the methods. To evaluate the preventive and therapeutic effects of dimerized HRF/TCTP-binding peptide (dTBP2) in the CIA model, dTBP2 was prepared as described in the methods. Male DBA/1 mice (7-9 weeks old) were immunized with $100 \mu \mathrm{g}$ of bovine type II collagen (BII; Chondrex) emulsified with an equal 
volume of $2 \mathrm{mg} / \mathrm{mL}$ CFA. Immunization was boosted with an equal amount of BII emulsified in IFA on day 21. In the preventive approach, CIA mice were administered daily i.p. injections of the vehicle or dTBP2 $(5,12.5$, or $25 \mathrm{mg} / \mathrm{kg})$ from Day 21 until the end of the experiment. In the therapeutic approach, following the development of overt arthritis (average score of 5) on day 41, CIA mice were administered daily i.p. injections of the vehicle or dTBP2 ( 5 or $25 \mathrm{mg} / \mathrm{kg}$ ) from Day 41 until the end of the experiment.

Generation of TCTP knockout (TCTP+/-) and transgenic (TG) mice

TCTP-TG mice were generated in the C57BL/6 and CBA hybrid background, using a targeting construct pCAGGS-TCTP cDNA containing the CMV-IE and chicken $\beta$-actin promoter as previously described (Chen et al., 2007), they were then backcrossed with C57BL/6 mice. Mice heterozygous for the deleted allele of TCTP, TCTP+/-, were kindly provided by Hsin-Fang Yang-Yen (Institute of Molecular Biology, Academia Sinica, Taipei, Taiwan) After the construction and electroporation of the targeting vector into the R1 ES cells, the positive clones were transiently transfected with a cytomegalovirus promoter-driven Cre expression vector, to obtain subclones of either the floxed or deleted TCTP allele. The ES cell clones so obtained were microinjected into mouse blastocysts and were backcrossed to generate $\mathrm{TCTP}+/$ - mice.

Arthritis score and ankle thickness measurement

Clinical arthritis scores were evaluated on a scale of $0-4$ for each limb $(0=$ no swelling; $1=$ slight swelling and erythema; $2=$ moderate swelling and erythema; $3=$ severe swelling and erythema; and $4=$ maximal inflammation with joint rigidity. The maximum possible score for each mouse was 16 . Hind paw thickness was measured using an electric caliper placed across the ankle joint at the widest point.

Micro-computed tomography (micro-CT) imaging

A SkyScan 1076 micro-CT apparatus (Kontich, Belgium) was used to evaluate structural changes in the ankle joints of CIA mice. Ankle joints were scanned and reconstructed into a 3-dimensional structure with a voxel size of $18 \mu \mathrm{m}$. The projections were reconstructed into 3-dimensional images using NRecon software (version 1.6.1.5) and CT Analyzer (version 1.10.0.1) (both from SkyScan).

Preparation of dimerized $H R F / T C T P(d-H / T)$ and dimerized HRF/TCTP-binding peptide

d-H/T was prepared as described previously (M. Kim et al., 2009). In brief, Escherichia coli BL21 (DE3) pLysS cells were transformed using pRSET A/mutated d-H/F, and the overexpressed d-H/F was then purified using a Ni2 ${ }^{+}$-charged His-Bind column according, to the manufacturer's protocol (ELPIS, Daejeon, Korea). NH2-terminal fusion proteins of $\mathrm{d}-\mathrm{H} / \mathrm{T}$ were separated by fast protein liquid chromatography on a HiTrap Q column (GE Healthcare, Piscataway, NJ, USA) using an $\mathrm{NaCl}$ gradient. Synthetic dTBP2, purified to over $98 \%$ purity, was provided by A \& PEP Inc.

\section{Isolation and culture of synovial fibroblasts}

Fibroblast-like synoviocytes (FLS) were isolated from synovial tissues of ten RA or three OA patients during total knee replacement surgery. The FLSs of OA patients were used as a control. The synovial tissues were minced and treated for $4 \mathrm{~h}$ with $2.5 \mathrm{mg} / \mathrm{mL}$ of type I collagenase (Sigma-Aldrich, Saint Louis, MO) in Dulbecco's modified Eagle's medium (DMEM) at $37^{\circ} \mathrm{C}$ in $5 \% \mathrm{CO}_{2}$. Dissociated cells were then centrifuged at $1000 \times \mathrm{g}$, resuspended in DMEM supplemented with $10 \%$ fetal bovine serum (FBS) and $2.5 \mu \mathrm{g} / \mathrm{ml}$ of amphotericin B, and seeded in $75 \mathrm{~cm}^{2}$ flasks (BD Biosciences, Franklin Lakes, NJ). After overnight culture, nonadherent cells were removed, and adherent cells were cultured in DMEM supplemented with 10\% FBS. The cultures were maintained at $37^{\circ} \mathrm{C}$ in $5 \% \mathrm{CO}_{2}$, and the medium was replaced every $3 \mathrm{~d}$. The purity of the cells was tested by flow-cytometric analysis using fluorescein isothiocyanate phycoerythrin-conjugated antiCD3 (Thermo Fisher Scientific Cat\# 11-0037-42, RRID:AB_2016669), anti-CD14 (Thermo Fisher Scientific Cat\# 11-0149-42, RRID:AB_10597597 ), anti-CD19 (Thermo Fisher Scientific Cat\# 11-0199-42, RRID:AB_10669461), or anti-CD90 (BD Biosciences Cat\# 555595, RRID:AB_395969) monoclonal antibodies. A FACS Calibur flow cytometer (Becton Dickinson, San Jose, CA) was used for the analysis. Among ten RA-FLSs from different donors, we selected 3 , and the cells from passages 3 to 8 were used for each experiment. RA 
or OA FLSs were cultured with $1 \mathrm{ng} / \mathrm{ml}$ IL-1 $\beta$ (R\&D Systems), $75 \mathrm{nM}$ dTCTP, $75 \mathrm{nM}$ dTBP2, or $75 \mathrm{nM}$ dTCTP plus $75 \mathrm{nM}$ dTBP2 for $24 \mathrm{~h}$, and the supernatants were harvested. All treatments were performed in serum-free medium.

\section{Culture of FLS and Cell proliferation assay}

FLS were obtained from the synovial tissues of patients with RA or OA, as described previously (Hah et al., 2010). Dimerized HRF/TCTP (d-H/T) was prepared as described previously (M. Kim et al., 2009), and as described in the Supplementary Methods. RA-FLS were seeded at $5 \times 103$ cells/well in 96-well plates. After a $72 \mathrm{~h}$ incubation, the media were replaced with DMEM containing $0.1 \%$ fetal bovine serum (FBS) supplemented with $75 \mathrm{nM}$ dH/T plus $75 \mathrm{nM}$ dTBP2. The rate of cell proliferation was measured using Cell Counting Kit-8 (CCK-8; Dojindo Laboratories, Kumamoto, Japan) according to the manufacturer's instruction.

\section{Wound closure and cell invasion assay}

Wound closure and cell invasion assays were performed as described previously (Jeon et al., 2017). In brief, RA-FLS monolayers, grown to confluence on $60-\mathrm{mm}$ culture dishes, were scratched with a sterile $200-\mu \mathrm{l}$ pipette tip and then treated with $75 \mathrm{nM} \mathrm{dH} / \mathrm{T} 2$, or $75 \mathrm{nM} \mathrm{dH} / \mathrm{T}$ plus $75 \mathrm{nM} \mathrm{dTBP} 2$. The width of the wounded area was imaged and measured under an inverted phase-contrast microscope (Nikon) at 50× magnification, to assess cell migration at 0 and $48 \mathrm{~h}$ after scratching. To examine cell invasiveness, Transwell migration assay chambers (BD, Franklin Lakes, NJ, USA) with an 8- $\mu \mathrm{m}$ pore size were used for a two-chamber migration assay. The upper surfaces of transwell inserts were coated with Matrigel (BD; $50 \mathrm{mg} /$ filter), and RA-FLS were seeded using serum-free medium. Complete growth medium supplemented with dH/T (75 $\mathrm{nM}$ ), or with $75 \mathrm{nM} \mathrm{dH} / \mathrm{T}$ plus $75 \mathrm{nM}$ dTBP2, was used as a chemoattractant in the lower chamber. After $24 \mathrm{~h}$, cells that invaded through the bottom surface of the insert were fixed with $10 \%$ formalin solution, stained with propidium iodide (Invitrogen), and quantified by counting the number of cells in 10 randomly selected viewing fields.

\section{$R N A$ isolation and quantitative real-time ( $q R T)-P C R$}

Total RNA, extracted from frozen joint tissues using TRIzol (Invitrogen, Carlsbad, CA, USA), was subjected to cDNA synthesis using the iScript cDNA synthesis kit (Bio-Rad, Hercules, CA, USA), as per manufacturer's instructions. qRT-PCR was performed on a ViiA 7 Real-time PCR System (Applied Biosystems, Foster City, CA) using TaqMan Universal PCR Master Mix (Applied Biosystems) and TaqMan primer/probe sets (PreDeveloped Assay Reagents; Applied Biosystems) for IL-13, IL-6, MMP-1, MMP-3, MMP13, and CCR2 (all as per the manufacturers' instructions). Data were normalized to the expression of GAPDH (internal control), and relative expressions were calculated using the $2^{-\mathrm{Ct}}$ method.

\section{Histopathological examination}

Ankle joint tissues were harvested, fixed in 10\% formalin, decalcified for 4 weeks in 10\% EDTA, embedded in paraffin, and sectioned into $5 \mu \mathrm{m}$ sections. The sections were then stained with hematoxylin and eosin (H\&E), safranin-O, or tartrate-resistant acid phosphatase (TRAP). Synovial inflammation, cartilage damage, and bone erosion were evaluated and TRAP-positive multinucleated osteoclasts (with [?] 3 nuclei) were counted as described previously (Jeon et al., 2017).

\section{Immunohistochemical and immunofluorescent staining}

For immunohistochemical staining, human synovial tissues sections were incubated with an anti-HRF/TCTP antibody (1:100; Abcam Cat\# ab133568, RRID:AB_2833070) overnight at $4^{\circ} \mathrm{C}$. Sections were then incubated with biotinylated anti-rabbit IgG (Vector Laboratories) for $1 \mathrm{~h}$ at room temperature. HRF/TCTP-positive cells were detected using a Peroxidase-Conjugated Avidin-Biotin Complex (ABC) kit (Vector) followed by staining with 3'3-diaminobenzidine tetrahydrochloride (DAB, Sigma-Aldrich). For immunofluorescent staining, tissue sections were incubated with monoclonal anti-CD55 antibody (1:100; Santa Cruz Biotechnology Cat\# sc-51733, RRID:AB_629151) plus anti-HRF/TCTP antibody (1:100; Abcam Cat\# ab133568, RRID: 
RRID:AB_2833070) overnight at 4degC. Each slide was then incubated with Alexa Fluor 594-conjugated antirabbit IgG (Thermo Fisher Scientific Cat\# A-21207, RRID:AB_14163) or Alexa 488-conjugated anti-mouse IgG (Thermo Fisher Scientific Cat\# A327661, RRID:AB_2762823). Images of fluorescence were obtained using a fluorescence microscope (BX 51, Olympus) at excitation/emission wavelengths of 488/520 nm (for Alexa Fluor 488) or 594/617 nm (for Alexa Fluor 594).

Enzyme-linked immunosorbent assay (ELISA)

The levels of HRF/TCTP (LSBio, Seattle, WA), IgG, IgG1, and IgG2a (R\&D Systems, Minneapolis, MN, USA) in the sera, synovial fluid, and FLS culture supernatants were measured using ELISA kits, according to the instructions of the respective manufacturers.

\section{Western blot analysis}

Total proteins were extracted from FLS with RIPA lysis buffer supplemented with protease inhibitor cocktail (Calbiochem, San Diego, CA, USA). The nuclear fractionation were performed using NE-PER Nuclear and Cytoplasmic Extraction Reagents (Thermo Fisher Scientific). Total protein lysates or nuclear extracts were separated by SDS-PAGE, and transferred onto a membrane. For dimerized HRF/TCTP detection, proteins were fractionated by non-reducing SDS-PAGE. The membranes were blocked with $5 \%$ skimmed milk and incubated with the following primary antibodies: HRF/TCTP (Abcam Cat\# ab133568, RRID: RRID:AB_2833070), $\beta$-actin (GenDEPOT, Cat\# A0042, RRID:AB_2848168), ERK (Cell Signaling Technology Cat\# 9102, RRID:AB_330744), Phospho-ERK (Cell Signaling Technology Cat\# 9106, RRID:AB_331768), Phospho-I $\chi \mathrm{B} \alpha$ (Cell Signaling Technology Cat\# 2859, RRID:AB_561111), GAPDH (ABFrontier Cat\# LF-PA0018, RRID:AB_1616734), p65 (Enzo Life Sciences Cat\# ALX-210-574, RRID:AB_2153516) and Lamin A/C (Cell Signaling Technology Cat\# 2032, RRID:AB_2136278). Signals were visualized using the corresponding HRP-conjugated secondary antibodies and substrates. Bands were normalized using loading control and quantified by densitometry using Image J 1.48 software.

Mycobacterium tuberculosis (Mtb) culture

Mtb H37Rv (ATCC 27294) was purchased from the American Type Culture Collection (ATCC, Manassas, VA, USA). The original stock was streaked on a plate with OADC added onto 7H11 and incubated at 37 ${ }^{\circ} \mathrm{C}$. After 3-4 weeks, a single colony was picked and incubated for 2 weeks in a culture flask containing 7H9 broth supplemented with OADC. To this culture flask, 7H9 broth containing OADC was added, then the flask was incubated at $37^{\circ} \mathrm{C}$ for 7-10 days. The flask was incubated for another 7-10 days, after which single cells were prepared. The seed lots of each strain were stored in small aliquots at $-80 \operatorname{deg} \mathrm{C}$ until use.

\section{Mtb infection and treatments}

Specific pathogen-free 6-week-old C57BL/6J female mice were purchased from Japan SLC, Inc. (Shijuoka, Japan), and were strictly maintained under barrier conditions in a Biosafety Level 3 (BSL-3) facility at the Avison Biomedical Research Center, Yonsei University College of Medicine (Seoul, Korea). All animal studies were performed in accordance with the Korean Food and Drug Administration (KFDA) guidelines. The experimental protocols used in this study were approved by the Ethics Committee and Institutional Animal Care and Use Committee of the Laboratory Animal Research Center at Yonsei University College of Medicine (permit number: 2017-0342).

Mice were infected with Mtb strain H37Rv via aerosol (W. S. Kim et al., 2016). Mice were exposed to the Mtb strain H37Rv for $60 \mathrm{~min}$ in the calibrated inhalation chamber of an airborne infection apparatus that delivers a predetermined Mtb dose (Glas-Col, Terre Haute, IN), and approximately 150 viable bacteria were delivered into the lungs. To confirm the initial bacterial burden, mice were euthanized one day later, and approximately 150 viable bacteria were delivered to the lungs of each mouse. Mouse anti-TNF- $\alpha$ monoclonal antibody, (MP6-XT22; Biolegend, San Diego, CA) was administered intraperitoneally in PBS (0.25 mg/mouse; 200 $\mu \mathrm{l} /$ mouse) for neutralization, once before infection and once every week for 4 weeks after Mtb infection. dTBP $(50 \mathrm{mg} / \mathrm{kg}$ ) was administrated intraperitoneally in PBS (200 $\mu \mathrm{l} /$ mouse) twice per week for 4 weeks after infection. All mice were euthanized $30 \mathrm{~d}$ after Mtb infection. 


\section{Histopathological analysis and enumeration of $M t b$}

Disease phenotype and severity were evaluated through histopathology and by measuring bacterial growth in the lung and spleen. The organs were removed to determine how well they had been protected, at $30 \mathrm{~d}$ after infection. For lung histopathology, the right-superior lobes were fixed overnight in $10 \%$ formalin, and embedded in paraffin. Lungs were sectioned at $4-5 \mu \mathrm{m}$, and H\&E stained. For bacterial growth analysis, the lung and spleen were homogenized and serially diluted samples were plated onto Middlebrook 7H10 agar plates (Becton Dickinson, Franklin Lakes, NJ) supplemented with 10\% OADC (Difco Laboratories, Detroit, VA), $2 \mu \mathrm{g} / \mathrm{ml}$ of 2-thiophenecarboxylic acid hydrazide (Sigma-Aldrich, St. Louis, MO) and amphotericin B (Sigma-Aldrich). After 4 weeks of incubation at $37^{\circ} \mathrm{C}$, bacterial colonies were counted. Data are presented as $\log _{10}$ colony forming units (CFU) per organ.

\section{Statistical analysis}

Data were analyzed using two-way analysis of variance (ANOVA) followed by Fisher's post-hoc analysis. The significance of differences between groups was determined using a Student's unpaired $t$-test or one-way ANOVA with Tukey's post-hoc test. Data are expressed as means \pm SEM and a $p$-value of less than 0.05 was considered statistically significant.

\section{Results}

HRF/TCTP overexpression and knockdown are correlated with arthritic phenotypes in mice with CIA

We established the CIA model in TG mice to examine the role of HRF/TCTP overexpression in RA. CIA was significantly more severe in TG mice than littermate WT mice (Figure 1A). Assessment of joint pathology revealed that joint inflammation, bone erosion, and cartilage destruction in TG mice were more pronounced than those in WT mice(Figure 1B and 1C). We then examined whether HRF/TCTP knockdown would exert an anti-arthritic effect upon CIA generation. As we expected, KD mice showed an attenuated arthritic phenotype with respect to the WT mice (Figure 1D) . Micro-CT and pathological data demonstrated reduced bone destruction in KD mice compared with WT mice(Figure 1E). Similarly, synovial inflammation, bone erosion, cartilage damage, and osteoclastic bone resorption were less pronounced in KD mice compared with WT mice (Figure 1G-1I). These results show that HRF/TCTP enhanced the manifestation of CIA, but knockdown of HRF/TCTP inhibited the arthritic inflammation and joint destruction observed in CIA.

\section{$H R F / T C T P$ is highly expressed and positively correlated with disease activity in patients with RA}

To further determine the role of HRF/TCTP in human RA, we next assessed HRF/TCTP levels in human blood and joint fluid. While HRF/TCTP levels in other inflammatory diseases are similar to those observed in healthy individuals and patients with osteoarthritis (OA), only patients with advanced RA exhibited high serum levels of HRF/TCTP in our present study(Figure 2A). Additionally, HRF/TCTP levels were positively correlated with DAS28-CRP, DAS28-ESR, and serum levels of IL-6 in patients with RA (Figure 2B-2D) . Furthermore, HRF/TCTP levels in joint fluid were higher in patients in early stages of RA, and significantly higher in patients with advanced RA, than in patients with $\mathrm{OA}$ and healthy individuals employed as controls (Figure 2E) . Because the concentration of HRF/TCTP in joint fluid was 10-fold greater than in serum, we hypothesized that cells mainly responsible for secreting HRF/TCTP reside in the joint. Thus, we examined HRF/TCTP expression in human joint tissues. We found that expression of HRF/TCTP was higher in the RA synovium than in OA synovium. This finding is supported by the fact that CD55-positive RA-FLS, which represent the predominant cell type in RA synovium, were co-stained with HRF/TCTP(Figure 2F). The expression of HRF/TCTP, and particularly the active dimer form of HRF/TCTP (d-H/T), was higher in RA-FLS than in OA-FLS, and was further increased by stimulation with IL-1 $\beta$ (Figure $\mathbf{2 G}$ and $\mathbf{2 H}$ ) . Taken together, these results indicate that RA-FLS, stimulated by the inflammatory environment, can robustly secrete HRF/TCTP. This result explains why levels of HRF/TCTP were higher, and correlated well with clinical disease activity, in patients with RA.

$H R F / T C T P$ activates promigratory and invasive phenotypes of $R A-F L S$ 
Next, we examined the effects of HRF/TCTP on the cancer-like phenotype of RA-FLS. Our results show that $\mathrm{d}-\mathrm{H} / \mathrm{T}$, as an active dimerized form of HRF/TCTP, stimulated the migration, invasiveness, and proliferation of RA-FLS. Blocking d-H/T via dTBP2, as an HRF/TCTP-binding peptide, decreased the aggressiveness of d-H/T-stimulated RA-FLS (Figure 3A-C, F) . Treatment with $\mathrm{d}-\mathrm{H} / \mathrm{T}$ enhanced the expression of inflammatory cytokines, including IL-1 $\beta$, IL-6, and MMP1, while treatment with dTBP2 abolished increased expression of these cytokines (Figure 3D-E). We evaluated the effect of HRF/TCTP on pathways involving RA-FLS, d-H/T-induced increases in ERK phosphorylation in the mitogen-activated protein kinase (MAPK) pathway, phosphorylation of $\mathrm{I} \chi \mathrm{B} \alpha$, and nuclear translocation of $\mathrm{p} 65$ in the $\mathrm{NF} \varkappa \mathrm{B}$ pathway. As expected, these effects were blocked by dTBP2. Collectively, these results show that HRF/TCTP plays a crucial role in stimulating the cancer-like aggressiveness of RA-FLS via the ERK and p65 pathways.

$H R F / T C T P$ blockade prevents arthritic development in mice with CIA

Based on our in vitro data showing that dTBP2 dramatically inhibited the inflammatory and cancer-like characteristics of RA-FLS, we next evaluated whether dTBP2 can prevent the development of autoimmune arthritis in a CIA mouse model. Mice with CIA, treated with 12.5 or $25 \mathrm{mg} / \mathrm{kg}$ of dTBP2, showed a significantly decreased clinical score and ankle thickness compared to those injected with the vehicle(Figure $\mathbf{4 A}$ and 4B). Histological analyses of ankle joints harvested from mice treated with $25 \mathrm{mg} / \mathrm{kg}$ of dTBP2 showed milder arthritic characteristics, such as decreased joint inflammation, bone erosion, cartilage destruction, and osteoclastic bone resorption than those of mice treated with the vehicle (Figure 4C-E) . The expression levels of IL-1 $\beta$, IL-6, MMP-3, MMP-13, and CCR2 were significantly lower in CIA mice treated with 25 $\mathrm{mg} / \mathrm{kg}$ of dTBP2 than in vehicle-treated CIA mice (Figure $4 \mathbf{F}$ ). Total IgG, IgG1, and IgG2a concentrations in the sera of CIA mice treated with $25 \mathrm{mg} / \mathrm{kg}$ of dTBP2 were lower than that in vehicle-injected CIA mice (Figure 4G) . These results show that dTBP2 prevented the development of joint inflammation and destruction in CIA.

\section{$H R F / T C T P$ blockade exerts therapeutic effects in mice with CIA}

Next, we assessed the possible therapeutic effects of dTBP2 on the progression of arthritis. CIA mice (average arthritis score $=5$ ) were injected with the vehicle, or with 5 or $25 \mathrm{mg} / \mathrm{kg}$ of dTBP2. Mice treated with 5 $\mathrm{mg} / \mathrm{kg}$ of dTBP2 showed partially improved clinical scores until 54 weeks. In particular, CIA mice treated with $25 \mathrm{mg} / \mathrm{kg}$ dTBP2 showed a greater reduction in arthritic phenotype than mice treated with the vehicle or with $5 \mathrm{mg} / \mathrm{kg}$ dTBP2 (Figure 5A). Ankle thickness showed a sustained decrease in mice treated with 25 $\mathrm{mg} / \mathrm{kg}$ of dTBP2(Figure 5B) . Assessment of ankle joint pathology revealed that joint inflammation, bone erosion, cartilage destruction, and osteoclastic bone resorption were more attenuated in mice treated with $25 \mathrm{mg} / \mathrm{kg}$ of dTBP2 than in the vehicle-injected group (Figure 5C-5E) . Compared with vehicle-treated mice, levels of IL-1 $\beta$, IL-6, MMP-3, MMP-13, and CCR2 were significantly decreased in the joint tissues of mice treated with $25 \mathrm{mg} / \mathrm{kg}$ of dTBP2 (Figure $\mathbf{5 F}$ ). Consistent with these results, dTBP2 treatment led to decrease in the serum levels of total IgG, IgG1, and IgG2a (Figure 5G) . Furthermore, the reactivation of latent Mycobacterium tuberculosis (Mtb) infections is an important factor in the safety of RA therapeutics. In the Mtb infection and treatment experiments aimed at ruling out these clinical concerns, dTBP2 showed a lower severity of TB infection than the anti-TNF- $\alpha$ monoclonal antibody used in RA treatment and showed a similar phenotype to that of the vehicle-treated groups(Figure 6A-D) . Collectively, dTBP2 exerted preventive and therapeutic effects by downregulating the synthesis of numerous proinflammatory cytokines and antibodies, thereby slowing the progression of CIA without increased the risk of Mtb reactivation.

\section{Discussion}

In agreement with previous findings indicating that HRF/TCTP contributes to allergic inflammation and tumorigenesis (Acunzo et al., 2014; M. Kim, Maeng, \& Lee, 2013), we have shown in our present study the novel role and clinical implication of HRF/TCTP expression in the pathogenesis of RA. Out of multiple inflammatory diseases, HRF/TCTP is highly expressed only in RA, and its serum levels are correlated with RA disease activity. This result suggests that HRF/TCTP levels can be used as a biomarker in diagnosing and predicting the severity of RA. In RA, HRF/TCTP is mainly produced by RA-FLS, and plays a pivotal 
role in conferring RA-FLS with tumor-like capabilities, including increased migratory capacity, invasiveness, and enhanced proliferation. Experimental arthritis was promoted in HRF/TCTP TG mice and suppressed in KD mice. Inhibition of HRF/TCTP activity via dTBP2 shows preventive and therapeutic potential because it downregulates the synthesis of numerous proinflammatory cytokines and antibodies in CIA.

$\mathrm{HRF} / \mathrm{TCTP}$, as its name suggests, participates in histamine release and is mainly responsible for allergic diseases (Sampson et al., 1989; Warner et al., 1986; Yoneda et al., 2004). Interestingly, histamine is present in both the synovial tissue and joint fluid of patients with RA, and is recognized as one of the mediators of joint inflammation (Malone, Irani, Schwartz, Barrett, \& Metcalfe, 1986; Maslinska, Gujski, Laure-Kamionowska, Szukiewicz, \& Wojtecka-Lukasik, 2004; Woolley, 2003). Other studies, however, indicate that histamine levels are lower in the blood and joint fluid of patients with RA than in the controls. Additionally, prolonged treatment with anti-TNF- $\alpha$ significantly increases circulating levels of histamine, suggesting that histamine is not useful as a biomarker assessing RA (Adlesic et al., 2007). Conversely, a study using preclinical models of arthritis reported that both histamine H4 receptor (H4R)-deficient mice, and mice treated with an H4R antagonist, show reduced arthritic severity (Cowden et al., 2014). However, a clinical trial examining toreforant, a selective H4R antagonist, was terminated prematurely due to patient fatality and no significant alleviation of RA (Boyle et al., 2019; Thurmond et al., 2016). In contrast, HRF/TCTP exhibits cytokinelike upstream functions; while it can thus potentiate histamine release, it also induces IL- 8 and GM-CSF secretion, augments B-cell proliferation, and increases immunoglobulin (Yoneda et al., 2004). This upstream stimulatory activity supports the correlation of HRF/TCTP expression with the clinical and inflammatory RA activity observed in our study. The targeting of HRF/TCTP may also aid in guiding future successful therapeutic approaches for RA that are similar to the use of Syk and JAK inhibitors, which target upstream signaling cascade kinases inhibitors (Hammaker \& Firestein, 2010).

In humans, HRF/TCTP is generated by peripheral blood mononuclear cells, in addition to other cell types, such as T and B lymphocytes, platelets, vascular endothelial cells, and alveolar macrophages residing in tissue compartments (Thueson, Speck, Lett-Brown, \& Grant, 1979). Elevated levels of HRF/TCTP are found in the sera, bronchoalveolar lavage (BAL), and skin blister fluids of patients with asthma and various allergies (Warner et al., 1986; Yoneda et al., 2004). In our study, increased levels of HRF/TCTP were found in the sera and joint fluid of patients with RA. HRF/TCTP levels were approximately 10-fold higher in the joint fluid than in the sera, which is unexpected. This finding suggests that joint-stromal cells are the main source of HRF/TCTP in RA. Once activated, RA-FLS acquire robust proliferative capacity, demonstrate tumorlike behavior, and become the most abundant stromal cells in the inflamed joint synovium. Consistent with a previous report showing the presence of HRF/TCTP in the synovial pannus and FLS of patients with RA (27), our study also shows that HRF/TCTP expression was higher in the synovium and FLS of patients with RA than in those of patients with OA. Furthermore, our results show that IL- $1 \beta$ stimulation increased RA-FLS production of HRF/TCTP. In agreement with previous reports, our results demonstrate that HRF/TCTP was generated mainly by RA-FLS.

Our previous studies, as well as those of others, have recently demonstrated the presence of a dimerized form of HRF/TCTP in the sera and BAL fluids of patients and mice with atopy and asthma; additionally, dimerization appears to be essential for the proinflammatory activity of HRF/TCTP in sites of inflammation (M. Kim et al., 2009). The results of our present study show that after stimulation with IL-1 $\beta$, levels of dimerized HRF/TCTP were increased, and this active form drove the transformation of RA-FLS into the aggressive inflammatory phenotype. MAPK and nuclear factor $\chi \mathrm{B}(\mathrm{NF} \varkappa \mathrm{B})$ are involved in regulating the aggressiveness of RA-FLS (Bottini \& Firestein, 2013). Our previous study demonstrated that dimerized $\mathrm{HRF} / \mathrm{TCTP}$ induces increased expression of IL-8, an inflammatory cytokine, via MAPK and NF- $x \mathrm{~B}$ signaling in human bronchial epithelial cells (45). In our present study, dimerized HRF/TCTP increased the phosphorylation of ERK and $\mathrm{I} \varkappa \mathrm{B} \alpha$, and increased nuclear translocation of $\mathrm{p} 65$ in the $\mathrm{NF} \varkappa \mathrm{B}$ pathway. Thus, consistent with previous studies highlighting the importance of HRF/TCTP activity in cancer cells (Acunzo et al., 2014), our results indicate a link between HRF/TCTP and the cancer-like behavior of RA-FLS, which crucially contributes to development and progression of RA. 
Because dimerized HRF/TCTP plays such a critical role in inflammatory diseases such as RA, blocking dimerized HRF/TCTP may offer a rational strategy for the treatment of RA. For this reason, we previously searched for dimerized HRF/TCTP-binding peptides (dTBPs) by screening a phage-displayed 7-mer peptide library. One peptide, designated as dTBP2, showed a stronger affinity for dimerized HRF/TCTP than for the inactive monomer form, and dTBP2 alleviated several allergy-related symptoms in animal models of rhinitis (M. Kim et al., 2013). In our present study, dTBP2 markedly inhibited the aggressiveness of RA-FLS exacerbated by dimerized HRF/TCTP. In both the preventive and therapeutic approaches, dTBP2 robustly blocked the development and progression of arthritis in mice with CIA. The CIA model offers an opportunity to evaluate preventive treatments in preclinical stages of RA, since prevention of RA is rather difficult to study in humans (Dekkers, Schoones, Huizinga, Toes, \& van der Helm-van Mil, 2017). In assessing the effectiveness of new drug candidates in animal RA models, a therapeutic, rather than preventive, approach is more predictive of a drug's clinical efficacy in human patients with RA (Bevaart, Vervoordeldonk, \& Tak, 2010; Hegen, Keith, Collins, \& Nickerson-Nutter, 2008). In our present study, dTBP2 exerted protective and therapeutic effects in CIA mice. Thus, our results strongly suggest that dTBP2 is an attractive new strategy for the prevention and treatment of RA.

In summary, our study shows that HRF/TCTP levels were increased in samples obtained from patients with RA, and correlated well with RA disease activity. HRF/TCTP was generated mainly by RA-FLS, and the active dimer form of HRF/TCTP drove RA-FLS toward a cancer-like phenotype. Consistent with these in vitro findings, HRF/TCTP overexpression led to an exacerbation of arthritis, while HRF/TCTP knockdown attenuated arthritic manifestations in mice with CIA. dTBP2, a blocking peptide of HRF/TCTP, abolished the pathologic effects of RA-FLS and dramatically inhibited the development and progression of arthritis in mice with CIA. Additionally, dTBP2 did not aggravate Mtb, while treatment with anti-TNF- $\alpha \mathrm{mAb}$ aggravated Mtb by causing a significant increase in bacterial burden along with excessive lung inflammation in a murine Mtb model. Together, our findings suggest that HRF/TCTP is an attractive target and that dTBP2 has preventive and therapeutic applications in patients with RA.

\section{Funding sources}

This work was supported by grants (HI17C0631) from the Korea Health Industry Development Institute (KHIDI) funded by the Ministry of Health \& Welfare (https://www.khidi.or.kr/kps) and (NRF2015R1A5A2008833) from the National Research Foundation of Korea (NRF) (http://www.nrf.re.kr/index) funded by the Korean government.

\section{Author contributions}

M.K., Y.C., H.L., K.L., and S.L. conceived the project and designed the experiments. M.K., Y.C., H.L. M.G.J., J.H.P., H.S.N., Y.C., H.J.P., J.P., and S.J.S. performed the experiments, interpreted the data, and helped with the discussion of the results. K.L. provided essential materials and helped with the discussion of the results. M.K., Y.C., H.L drafted the paper. K.L. and S.L. revised the paper. All authors commented on the manuscript.

\section{References}

Acunzo, J., Baylot, V., So, A., \& Rocchi, P. (2014). TCTP as therapeutic target in cancers. Cancer Treat Rev, 40 (6), 760-769. doi:10.1016/j.ctrv.2014.02.007

Adlesic, M., Verdrengh, M., Bokarewa, M., Dahlberg, L., Foster, S. J., \& Tarkowski, A. (2007). Histamine in rheumatoid arthritis. Scand J Immunol, 65 (6), 530-537. doi:10.1111/j.1365-3083.2007.01938.x

Aletaha, D., Neogi, T., Silman, A. J., Funovits, J., Felson, D. T., Bingham, C. O., 3rd, . . . Hawker, G. (2010). 2010 Rheumatoid arthritis classification criteria: an American College of Rheumatology/European League Against Rheumatism collaborative initiative. Arthritis Rheum, 62 (9), 2569-2581. doi:10.1002/art.27584

Altman, R., Asch, E., Bloch, D., Bole, G., Borenstein, D., Brandt, K., . . . et al. (1986). Development 
of criteria for the classification and reporting of osteoarthritis. Classification of osteoarthritis of the knee. Diagnostic and Therapeutic Criteria Committee of the American Rheumatism Association. Arthritis Rheum, 29 (8), 1039-1049. doi:10.1002/art.1780290816

Bae, S. Y., Kim, H. J., Lee, K. J., \& Lee, K. (2015). Translationally controlled tumor protein induces epithelial to mesenchymal transition and promotes cell migration, invasion and metastasis. Sci Rep, 5 , 8061. doi:10.1038/srep08061

Bevaart, L., Vervoordeldonk, M. J., \& Tak, P. P. (2010). Evaluation of therapeutic targets in animal models of arthritis: how does it relate to rheumatoid arthritis? Arthritis Rheum, 62 (8), 2192-2205. doi:10.1002/art.27503

Blum, A., \& Adawi, M. (2019). Rheumatoid arthritis (RA) and cardiovascular disease. Autoimmun Rev, 18 (7), 679-690. doi:10.1016/j.autrev.2019.05.005

Bottini, N., \& Firestein, G. S. (2013). Duality of fibroblast-like synoviocytes in RA: passive responders and imprinted aggressors. Nat Rev Rheumatol, 9 (1), 24-33. doi:10.1038/nrrheum.2012.190

Boyle, D. L., DePrimo, S. E., Calderon, C., Chen, D., Dunford, P. J., Barchuk, W., . . . Thurmond, R. L. (2019). Toreforant, an orally active histamine H4-receptor antagonist, in patients with active rheumatoid arthritis despite methotrexate: mechanism of action results from a phase 2, multicenter, randomized, doubleblind, placebo-controlled synovial biopsy study. Inflamm Res, 68 (4), 261-274. doi:10.1007/s00011-01901218-y

Bucala, R., Ritchlin, C., Winchester, R., \& Cerami, A. (1991). Constitutive production of inflammatory and mitogenic cytokines by rheumatoid synovial fibroblasts. $J$ Exp Med, 173 (3), 569-574. doi:10.1084/jem.173.3.569

Chen, S. H., Wu, P. S., Chou, C. H., Yan, Y. T., Liu, H., Weng, S. Y., \& Yang-Yen, H. F. (2007). A knockout mouse approach reveals that TCTP functions as an essential factor for cell proliferation and survival in a tissue- or cell type-specific manner. Mol Biol Cell, 18 (7), 2525-2532. doi:10.1091/mbc.e07-02-0188

Cheon, Y. H., Lee, S. G., Kim, M., Kim, H. O., Sun Suh, Y., Park, K. S., . . . Lee, S. I. (2018). The association of disease activity, pro-inflammatory cytokines, and neurotrophic factors with depression in patients with rheumatoid arthritis. Brain Behav Immun, 73 , 274-281. doi:10.1016/j.bbi.2018.05.012

Chitpatima, S. T., Makrides, S., Bandyopadhyay, R., \& Brawerman, G. (1988). Nucleotide sequence of a major messenger RNA for a 21 kilodalton polypeptide that is under translational control in mouse tumor cells.Nucleic Acids Res, 16 (5), 2350. doi:10.1093/nar/16.5.2350

Cowden, J. M., Yu, F., Banie, H., Farahani, M., Ling, P., Nguyen, S., . . . Thurmond, R. L. (2014). The histamine H4 receptor mediates inflammation and Th17 responses in preclinical models of arthritis. Ann Rheum Dis, 73 (3), 600-608. doi:10.1136/annrheumdis-2013-203832

Criteria for diagnosis of Behcet's disease. International Study Group for Behcet's Disease. (1990). Lancet, 335 (8697), 1078-1080.

De Cock, D., \& Hyrich, K. (2018). Malignancy and rheumatoid arthritis: Epidemiology, risk factors and management. Best Pract Res Clin Rheumatol, 32 (6), 869-886. doi:10.1016/j.berh.2019.03.011

Dekkers, J. S., Schoones, J. W., Huizinga, T. W., Toes, R. E., \& van der Helm-van Mil, A. H. (2017). Possibilities for preventive treatment in rheumatoid arthritis? Lessons from experimental animal models of arthritis: a systematic literature review and meta-analysis. Ann Rheum Dis, 76 (2), 458-467. doi:10.1136/annrheumdis-2016-209830

Favalli, E. G., Pregnolato, F., Biggioggero, M., Becciolini, A., Penatti, A. E., Marchesoni, A., \& Meroni, P. L. (2016). Twelve-Year Retention Rate of First-Line Tumor Necrosis Factor Inhibitors in Rheumatoid Arthritis: Real-Life Data From a Local Registry.Arthritis Care Res (Hoboken), 68 (4), 432-439. doi:10.1002/acr.22788 
Firestein, G. S. (2003). Evolving concepts of rheumatoid arthritis.Nature, 423 (6937), 356-361. doi:10.1038/nature01661

Fransen, J., \& van Riel, P. L. (2009). The Disease Activity Score and the EULAR response criteria. Rheum Dis Clin North Am, 35 (4), 745-757, vii-viii. doi:10.1016/j.rdc.2009.10.001

Hah, Y. S., Lee, Y. R., Jun, J. S., Lim, H. S., Kim, H. O., Jeong, Y. G., . . . Park, B. H. (2010). A20 suppresses inflammatory responses and bone destruction in human fibroblast-like synoviocytes and in mice with collagen-induced arthritis. Arthritis Rheum, 62 (8), 2313-2321. doi:10.1002/art.27545

Hammaker, D., \& Firestein, G. S. (2010). "Go upstream, young man": lessons learned from the p38 saga. Ann Rheum Dis, 69 Suppl 1 , i77-82. doi:10.1136/ard.2009.119479

Hegen, M., Keith, J. C., Jr., Collins, M., \& Nickerson-Nutter, C. L. (2008). Utility of animal models for identification of potential therapeutics for rheumatoid arthritis. Ann Rheum Dis, 67 (11), 1505-1515. doi:10.1136/ard.2007.076430

Jeon, M. G., Cheon, Y. H., Lim, H. S., Yi, S. M., Suh, Y. S., Kim, H. O., . . . Lee, S. I. (2017). Suppressive Effects of TSAHC in an Experimental Mouse Model and Fibroblast-Like Synoviocytes of Rheumatoid Arthritis. Inflammation, 40 (6), 1825-1835. doi:10.1007/s10753-017-0621-6

Kim, M., Maeng, J., \& Lee, K. (2013). Dimerization of TCTP and its clinical implications for allergy. Biochimie, 95 (4), 659-666. doi:10.1016/j.biochi.2012.10.007

Kim, M., Min, H. J., Won, H. Y., Park, H., Lee, J. C., Park, H. W., . . . Lee, K. (2009). Dimerization of translationally controlled tumor protein is essential for its cytokine-like activity. PLoS One, 4 (7), e6464. doi:10.1371/journal.pone.0006464

Kim, M. J., Kwon, J. S., Suh, S. H., Suh, J. K., Jung, J., Lee, S. N., . . . Lee, K. (2008). Transgenic overexpression of translationally controlled tumor protein induces systemic hypertension via repression of Na+,K+-ATPase. J Mol Cell Cardiol, 44 (1), 151-159. doi:10.1016/j.yjmcc.2007.09.017

Kim, W. S., Kim, J. S., Cha, S. B., Kim, H., Kwon, K. W., Kim, S. J., . . . Shin, S. J. (2016). Mycobacterium tuberculosis Rv3628 drives Th1-type T cell immunity via TLR2-mediated activation of dendritic cells and displays vaccine potential against the hyper-virulent Beijing K strain. Oncotarget, 7 (18), 24962-24982. doi:10.18632/oncotarget.8771

Lee, H. S., Woo, S. J., Koh, H. W., Ka, S. O., Zhou, L., Jang, K. Y., . . . Park, B. H. (2014). Regulation of apoptosis and inflammatory responses by insulin-like growth factor binding protein 3 in fibroblast-like synoviocytes and experimental animal models of rheumatoid arthritis. Arthritis Rheumatol, 66 (4), 863-873. doi:10.1002/art.38303

Lefevre, S., Knedla, A., Tennie, C., Kampmann, A., Wunrau, C., Dinser, R., . . . Neumann, E. (2009). Synovial fibroblasts spread rheumatoid arthritis to unaffected joints. Nat Med, 15 (12), 1414-1420. doi:10.1038/nm.2050

Malone, D. G., Irani, A. M., Schwartz, L. B., Barrett, K. E., \& Metcalfe, D. D. (1986). Mast cell numbers and histamine levels in synovial fluids from patients with diverse arthritides. Arthritis Rheum, 29 (8), 956-963. doi:10.1002/art.1780290803

Mankia, K., \& Emery, P. (2016). Preclinical Rheumatoid Arthritis: Progress Toward Prevention. Arthritis Rheumatol, 68 (4), 779-788. doi:10.1002/art.39603

Maslinska, D., Gujski, M., Laure-Kamionowska, M., Szukiewicz, D., \& Wojtecka-Lukasik, E. (2004). Subcellular localization of histamine in articular cartilage chondrocytes of rheumatoid arthritis patients.Inflamm Res, 53 Suppl 1 , S35-36. doi:10.1007/s00011-003-0316-3

Maslinska, D., Opertowska, J., Chabros, W., Maslinska, M., Grzybowska-Kowalczyk, A., Paradowska, A., . . . Wojtecka-Lukasik, E. (2008). Histamine releasing factor (HRF) in pannus of joints affected by rheumatoid 
arthritis. Inflamm Res, 57 Suppl 1, S61-62. doi:10.1007/s00011-007-0630-2

Noss, E. H., \& Brenner, M. B. (2008). The role and therapeutic implications of fibroblast-like synoviocytes in inflammation and cartilage erosion in rheumatoid arthritis. Immunol Rev, 223 , 252-270. doi:10.1111/j.1600065X.2008.00648.x

Orr, C., Vieira-Sousa, E., Boyle, D. L., Buch, M. H., Buckley, C. D., Canete, J. D., . . . Veale, D. J. (2017). Synovial tissue research: a state-of-the-art review. Nat Rev Rheumatol, 13 (10), 630. doi:10.1038/nrrheum.2017.161

Pitzalis, C., Kelly, S., \& Humby, F. (2013). New learnings on the pathophysiology of RA from synovial biopsies. Curr Opin Rheumatol, 25 (3), 334-344. doi:10.1097/BOR.0b013e32835fd8eb

Rho, S. B., Lee, J. H., Park, M. S., Byun, H. J., Kang, S., Seo, S. S., . . . Park, S. Y. (2011). Antiapoptotic protein TCTP controls the stability of the tumor suppressor p53. FEBS Lett, 585 (1), 29-35. doi:10.1016/j.febslet.2010.11.014

Rutherford, A. I., Subesinghe, S., Hyrich, K. L., \& Galloway, J. B. (2018). Serious infection across biologictreated patients with rheumatoid arthritis: results from the British Society for Rheumatology Biologics Register for Rheumatoid Arthritis. Ann Rheum Dis, 77 (6), 905-910. doi:10.1136/annrheumdis-2017-212825

Sampson, H. A., Broadbent, K. R., \& Bernhisel-Broadbent, J. (1989). Spontaneous release of histamine from basophils and histamine-releasing factor in patients with atopic dermatitis and food hypersensitivity. $N$ Engl J Med, 321 (4), 228-232. doi:10.1056/NEJM198907273210405

Singh, J. A., Saag, K. G., Bridges, S. L., Jr., Akl, E. A., Bannuru, R. R., Sullivan, M. C., . . . McAlindon, T. (2016). 2015 American College of Rheumatology Guideline for the Treatment of Rheumatoid Arthritis.Arthritis Rheumatol, 68 (1), 1-26. doi:10.1002/art.39480

Thueson, D. O., Speck, L. S., Lett-Brown, M. A., \& Grant, J. A. (1979). Histamine-releasing activity (HRA). I. Production by mitogen- or antigen-stimulated human mononuclear cells. J Immunol, 123 (2), 626-632.

Thurmond, R. L., Greenspan, A., Radziszewski, W., Xu, X. L., Miao, Y., Chen, B., . . . Smolen, J. S. (2016). Toreforant, A Histamine H4 Receptor Antagonist, in Patients with Active Rheumatoid Arthritis Despite Methotrexate Therapy: Results of 2 Phase II Studies. J Rheumatol, 43 (9), 1637-1642. doi:10.3899/jrheum.160164

van der Linden, S., Valkenburg, H. A., \& Cats, A. (1984). Evaluation of diagnostic criteria for ankylosing spondylitis. A proposal for modification of the New York criteria. Arthritis Rheum, 27 (4), 361-368. doi:10.1002/art.1780270401

van Steenbergen, H. W., da Silva, J. A. P., Huizinga, T. W. J., \& van der Helm-van Mil, A. H. M. (2018). Preventing progression from arthralgia to arthritis: targeting the right patients. Nat Rev Rheumatol, 14 (1), 32-41. doi:10.1038/nrrheum.2017.185

Warner, J. A., Pienkowski, M. M., Plaut, M., Norman, P. S., \& Lichtenstein, L. M. (1986). Identification of histamine releasing factor(s) in the late phase of cutaneous IgE-mediated reactions. J Immunol, 136 (7), 2583-2587.

Woo, S. J., Lee, S. M., Lim, H. S., Hah, Y. S., Jung, I. D., Park, Y. M., . . . Lee, S. I. (2016). Myeloid deletion of SIRT1 suppresses collagen-induced arthritis in mice by modulating dendritic cell maturation. Exp Mol Med, 48 , e221. doi:10.1038/emm.2015.124

Woo, S. J., Noh, H. S., Lee, N. Y., Cheon, Y. H., Yi, S. M., Jeon, H. M., . . . Park, B. H. (2018). Myeloid sirtuin 6 deficiency accelerates experimental rheumatoid arthritis by enhancing macrophage activation and infiltration into synovium. EBioMedicine, 38 , 228-237. doi:10.1016/j.ebiom.2018.11.005

Woolley, D. E. (2003). The mast cell in inflammatory arthritis. N Engl J Med, 348 (17), 1709-1711. doi:10.1056/NEJMcibr023206 
Yenofsky, R., Cereghini, S., Krowczynska, A., \& Brawerman, G. (1983). Regulation of mRNA utilization in mouse erythroleukemia cells induced to differentiate by exposure to dimethyl sulfoxide. Mol Cell Biol, 3 (7), 1197-1203. doi:10.1128/mcb.3.7.1197

Yoneda, K., Rokutan, K., Nakamura, Y., Yanagawa, H., Kondo-Teshima, S., \& Sone, S. (2004). Stimulation of human bronchial epithelial cells by IgE-dependent histamine-releasing factor. Am J Physiol Lung Cell Mol Physiol, 286 (1), L174-181. doi:10.1152/ajplung.00118.2003

You, S., Koh, J. H., Leng, L., Kim, W. U., \& Bucala, R. (2018). The Tumor-Like Phenotype of Rheumatoid Synovium: Molecular Profiling and Prospects for Precision Medicine. Arthritis Rheumatol, 70 (5), 637-652. doi:10.1002/art.40406

Figure legends

Figure 1. Role of HRF/TCTP in autoimmune arthritis. A : Mean clinical scores of HRF/TCTPoverexpressing transgenic (TG) and littermate wild-type (WT) mice after generation of collagen-induced arthritis (CIA) ( $\mathrm{n}=7$ mice for each group). B : Representative images of ankle joint tissue, harvested from TG and WT mice and stained with hematoxylin and eosin (H\&E), showing changes in tissue morphology typical of CIA (bar $=500 \mu \mathrm{m})$. C : Pathological scores, including those for synovial inflammation, cartilage damage, and bone erosion, for ankle joints of CIA mice $(n=14$ ankles for each group).D : Mean clinical scores of HRF/TCTP- knockdown (KD) and WT mice after CIA induction ( $\mathrm{n}=5$ mice for each group). $\mathbf{E}$ : Micro-CT images of hind paws (left), and percentage of bone volume (BV), and ratio of bone surface to bone volume (BS/BV) (right). $\mathbf{F}-\mathbf{H}:(\mathrm{F})$ Representative images of ankle joint tissue harvested from KD and WT mice and stained with H\&E and Safranin-O $($ bar $=500 \mu \mathrm{m})$, or immunolabeled for TRAP $($ bar $=$ $50 \mu \mathrm{m})$. (G) Pathological scores and $(\mathrm{H})$ number of TRAP-positive cells ( $\mathrm{n}=10$ ankles for each group). A high magnification view of the boxed area is presented in the right panel. Values are mean $\pm \mathrm{SEM} ;{ }^{*} \mathrm{p}<$ $0.05 ; * * \mathrm{p}<0.01{ }^{* * *} \mathrm{p}<0.001$ versus $\mathrm{WT}$.

Figure 2. Levels of HRF/TCTP in patients with rheumatoid arthritis (RA), and their relationship with disease severity. A : Levels of HRF/TCTP in the sera of healthy controls $(\mathrm{HC}, \mathrm{n}=15)$ and patients with osteoarthritis (OA, $\mathrm{n}=20$ ), ankylosing spondylitis (AS, $\mathrm{n}=23$ ), Behçet's disease (BD, $\mathrm{n}=26$ ), early RA (ERA, $\mathrm{n}=21$, disease duration $<1$ year), and advanced RA (ARA, $\mathrm{n}=17$, disease duration [?] 1 year) as determined by ELISA. B-D : Correlation of serum HRF/TCTP levels with (B) DAS28-CRP $(\mathrm{n}=62)$, (C) DAS28-ESR $(\mathrm{n}=62)$, and (D) serum IL-6 concentration of patients with RA $(\mathrm{n}=60)$. $\mathbf{E}$ : Levels of HRF/TCTP in the joint fluids of healthy controls $(\mathrm{HC}, \mathrm{n}=5)$ and patients with osteoarthritis $(\mathrm{OA}, \mathrm{n}=9$ ), early RA (ERA, $\mathrm{n}=6$ ), and advanced RA (ARA, $\mathrm{n}=12$ ). F: HRF/TCTP expression in synovial tissue of patients with OA and RA, determined by immunohistochemistry (left) and double immunofluorescence labeling (right) for CD55 (green) and HRF/TCTP (red). Scale bar: $100 \mu \mathrm{m}$. G: Protein expression of monomeric and dimeric forms of HRF/TCTP in OA-FLS, RA-FLS, and IL-1 $\beta$ stimulated RA-FLS, determined by western blotting (left); relative density, normalized to that of $\beta$-actin (right). Values are mean $\pm \mathrm{SEM} ;{ }^{*} \mathrm{p}<0.05 ;{ }^{* * *} \mathrm{p}<0.001$ versus healthy controls (A, E) or OA-FLS (H); \#\# $\mathrm{p}<0.01 ; \# \# \# \mathrm{p}<0.001$ versus before IL-1 $\beta$ stimulation $(\mathrm{H})$.

Figure 3. Effects of dimerized HRF/TCTP (d-H/F), and inhibitory effects of dimerized HRF/TCTP-binding peptide (dTBP2), on rheumatoid arthritis fibroblast-like synoviocytes (RA-FLS). RA-FLS treated with the vehicle, $75 \mathrm{nM} \mathrm{d-H/F}$, or $75 \mathrm{nM} \mathrm{d-H/F}$ plus $75 \mathrm{nM} \mathrm{dTBP2.} \mathrm{A} \mathrm{:}$ Representative microphotographs showing cells that had migrated into the wounded area after $48 \mathrm{~h}$ (upper panel, $50 \times$ magnification), and invasive cells that passed through the Matrigel-coated Transwell chamber after $24 \mathrm{~h}$ (lower panel, 100× magnification). B and C : (B) Migrated and (C) invading cells, quantified by counting in randomly selected fields.D and $\mathbf{E}$ : (D) Levels of inflammatory cytokines IL-1 $\beta$, IL-6, and MMP1 in RA-FLS treated with the vehicle, dTBP2, d-H/F, or d-H/F plus dTBP2, determined by qRT-PCR; (E) Production of IL-6, measured in culture supernatants using ELISA. F : Change in proliferation of RA-FLS treated with the vehicle, $\mathrm{d}-\mathrm{H} / \mathrm{F}$, or $\mathrm{d}-\mathrm{H} / \mathrm{F}$ with dTBP2, measured by WST-1 assay. G : Protein levels of ERK, phospho-ERK, and IkB in whole-cell extracts, and levels of p65 in nuclear fractions, determined by 
western blotting. GAPDH or lamin $\mathrm{A} / \mathrm{C}$ were used as internal controls. Values are mean $\pm \mathrm{SEM}$ of three experiments; ${ }^{*} \mathrm{p}<0.05 ;{ }^{* *} \mathrm{p}<0.01$; ${ }^{* * *} \mathrm{p}<0.001$ versus vehicle-treated RA-FLS; \# $\mathrm{p}<0.05$; \#\#\# $\mathrm{p}<$ 0.001 versus d-H/F-treated RA-FLS.

Figure 4. Prevention of inflammatory arthritis by dimerized HRF/TCTP-binding peptide (dTBP2). After generation of collagen-induced arthritis (CIA), the mice were treated with intraperitoneal injection of the vehicle or dTBP2, starting on day 21 (day of booster immunization) and continuing once every day until the day of sacrifice (day 41).A and B : (A) Mean clinical scores and (B) ankle thickness change of CIA mice treated with the vehicle or dTBP2 (at $5,12.5$, or $25 \mathrm{mg} / \mathrm{kg}$ ). C , Ankle tissues from groups treated with the vehicle or dTBP2 $(25 \mathrm{mg} / \mathrm{kg})$, stained using H\&E and safranin-O (bar $=500 \mu \mathrm{m})$, and immunolabeled for TRAP (bar $=50 \mu \mathrm{m})$. D and $\mathbf{E}:(\mathrm{D})$ Pathological scores for synovial inflammation, cartilage damage, and bone erosion, and (E) TRAP-positive cells in the ankle joints.F : Relative expression of inflammatory cytokines IL-1 $\beta$, IL-6, MMP3, MMP13, and CCR2 in ankle joints, determined by qRT-PCR.G : Levels of IgG, IgG1, and IgG2a in the sera of mice with CIA, quantified by ELISA. Values are mean \pm SEM (n: 10-12 mice for each group); ${ }^{*} \mathrm{p}<0.05 ;^{* *} \mathrm{p}<0.01 ;^{* * *} \mathrm{p}<0.001$ versus vehicle-treated CIA mice.

Figure 5. Therapeutic effects of dimerized HRF/TCTP-binding peptide (dTBP2) on inflammatory arthritis. After generation of collagen-induced arthritis (CIA), the mice were treated with intraperitoneal injections of the vehicle or dTBP2 starting on day 41 (the day when the average arthritis score was 5) and continuing once every day until the day of sacrifice (day 57). A and B : (A) Mean clinical score and (B) ankle thickness change of CIA mice treated with the vehicle or dTBP2 (at 5 or $25 \mathrm{mg} / \mathrm{kg}$ ). C : Ankle tissues from groups treated with the vehicle or dTBP2 $(25 \mathrm{mg} / \mathrm{kg}$ ), stained using H\&E and safranin-O (bar $=500 \mu \mathrm{m})$, and immunolabeled for TRAP $($ bar $=50 \mu \mathrm{m})$. D and E : (D) Pathological scores for synovial inflammation, cartilage damage, and bone erosion, and (E) TRAP-positive cells. F and G : (F) Relative expression of inflammatory cytokines in the ankle joint, and (G) levels of IgG, IgG1, and IgG2a in sera of vehicle- or dTBP2-treated CIA mice. Values are mean \pm SEM (n: 10-12 mice for each group); ${ }^{*} \mathrm{p}<0.05$; ** $\mathrm{p}<0.01$ versus vehicle-treated CIA mice.

Figure 6. dTBP does not reduce host protection against Mtb infection. Mice were infected with Mtb strain H37Rv via aerosolization with $150 \mathrm{CFU}$, and lungs were dissected $30 \mathrm{~d}$ post-infection for analysis.A: Changes in body weight were measured for up to $26 \mathrm{~d}$ after infection. B: The superior lobe of the right lung was stained with $\mathrm{H} \& \mathrm{E}$ at $30 \mathrm{~d}$ after Mtb challenge. $\mathbf{C}$ and $\mathbf{D}$ : The bacterial load in $(\mathrm{C})$ the lungs and (D) the spleens of each group at $30 \mathrm{~d}$ post-infection was analyzed by counting the bacteria (bar $=2$ $\mathrm{mm}$ ). Values are mean $\pm \mathrm{SD}$ (n: $5-6$ mice for each group); ${ }^{* *} \mathrm{p}<0.01 ;{ }^{* * *} \mathrm{p}<0.001$ versus vehicle-treated group. 
Figure 1

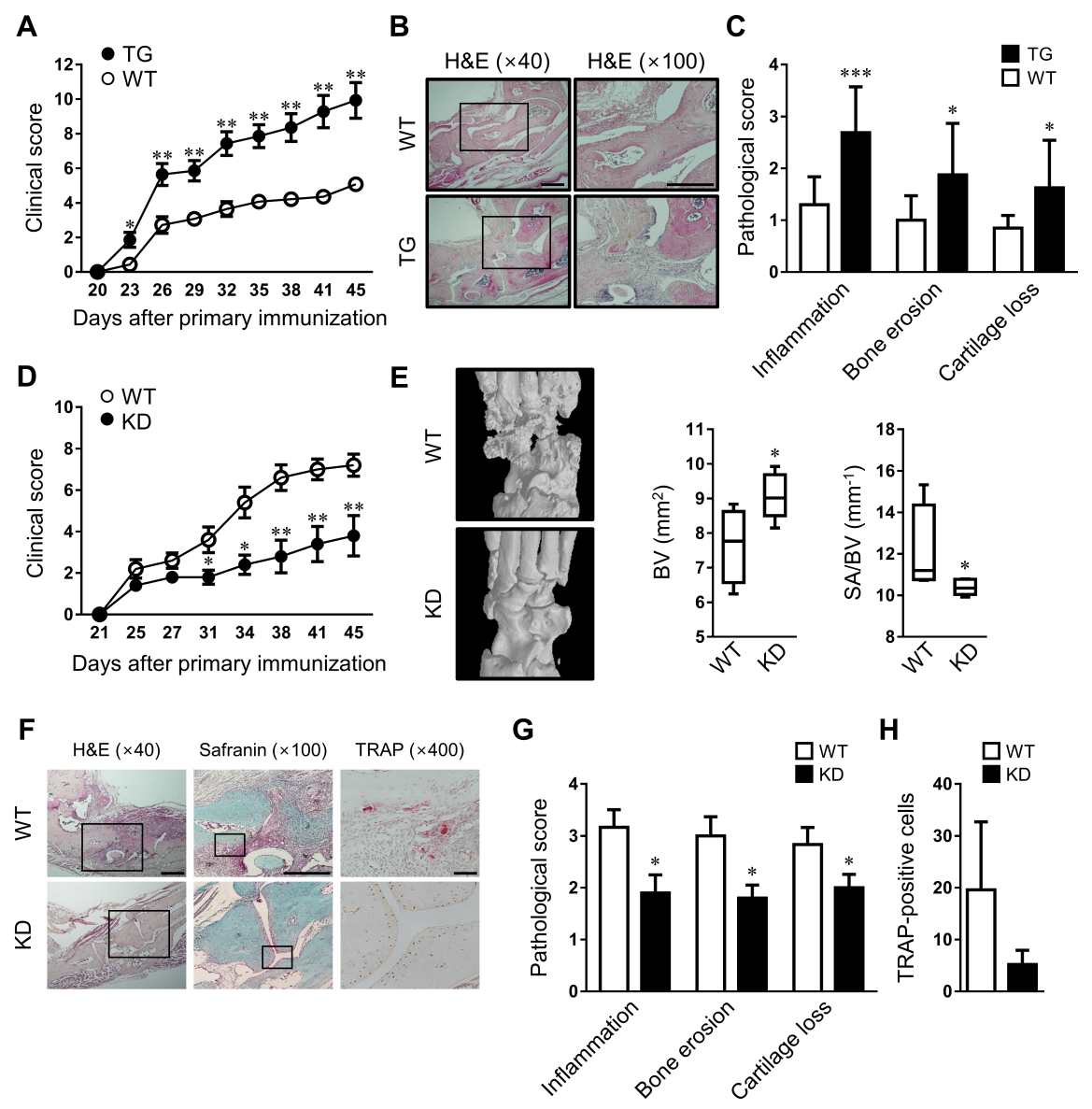


Figure 2
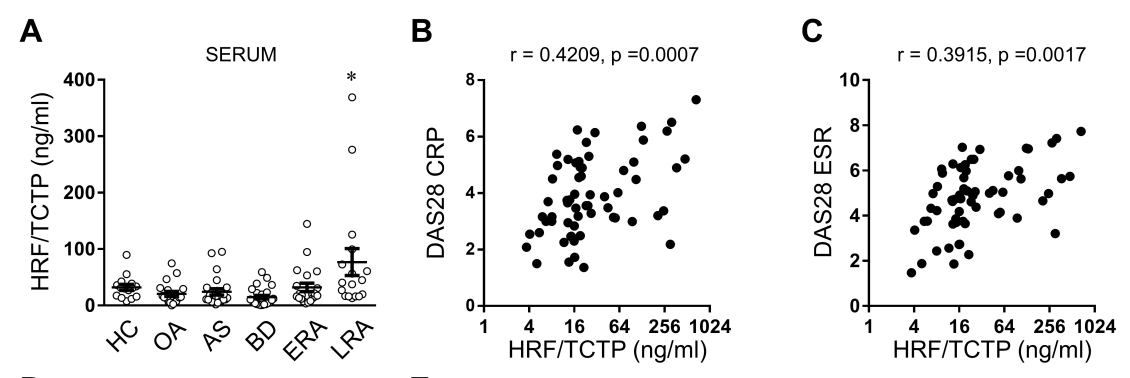

D

E
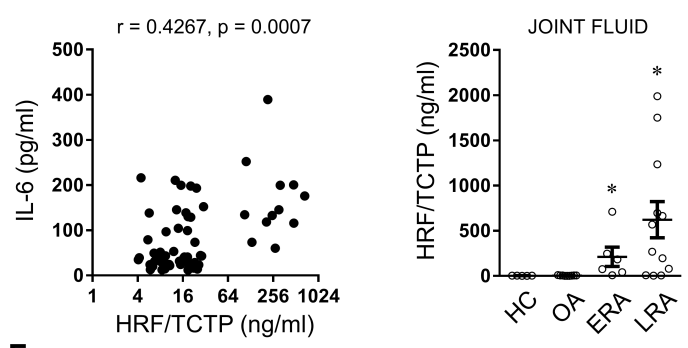

F
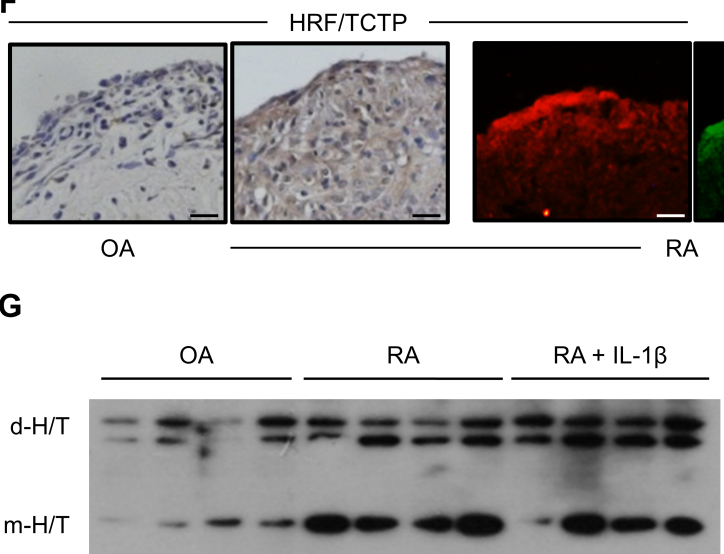

G

H $\quad$ ㅇ
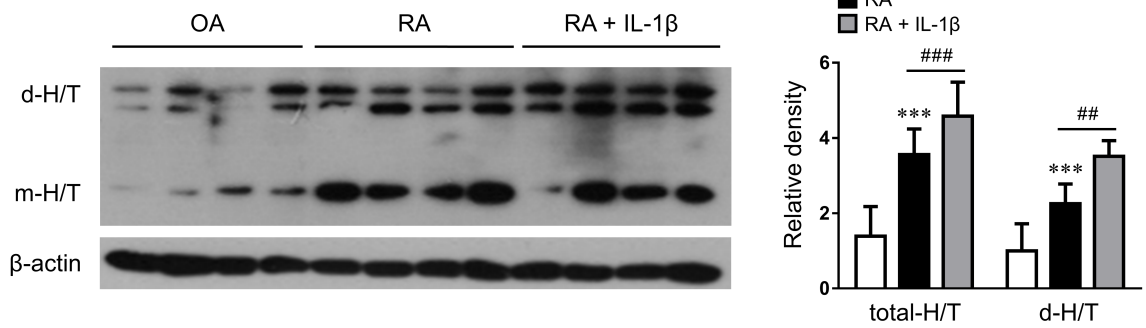
Figure 3
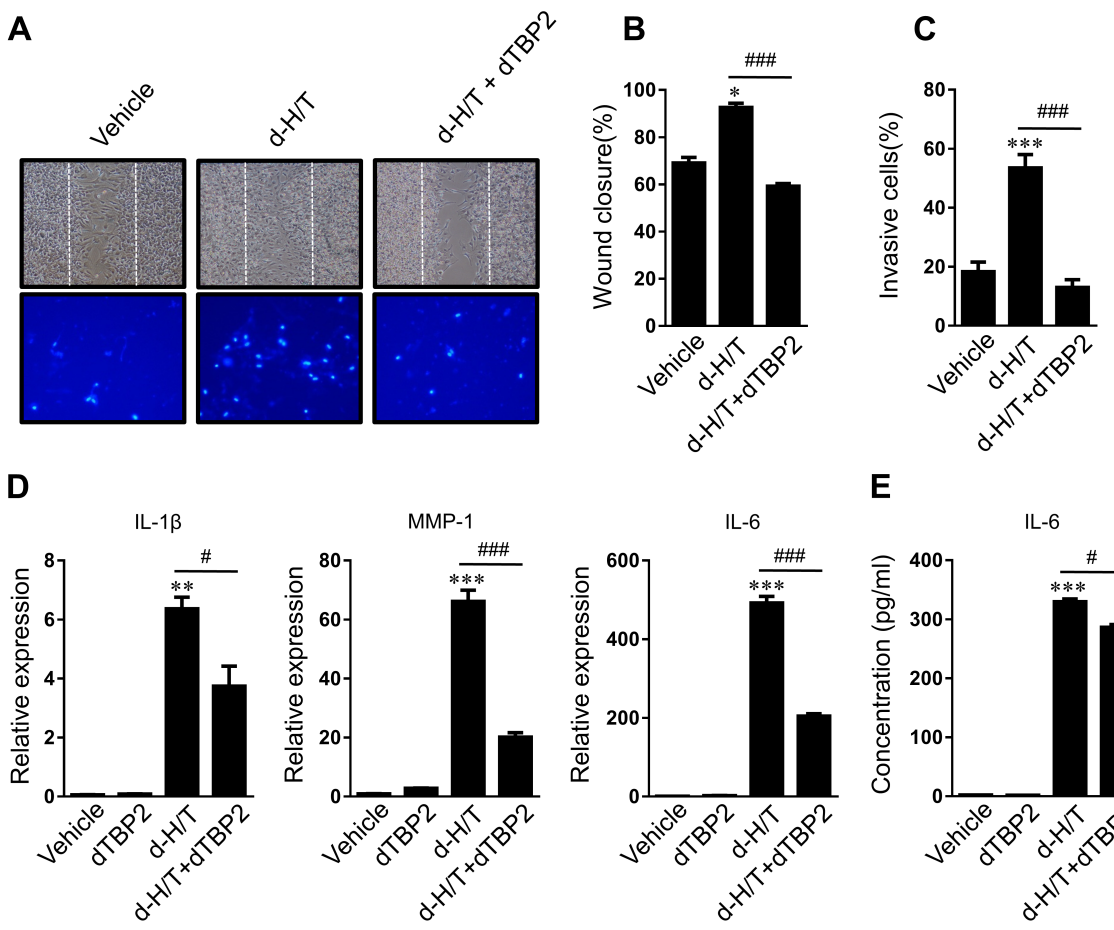

E

F

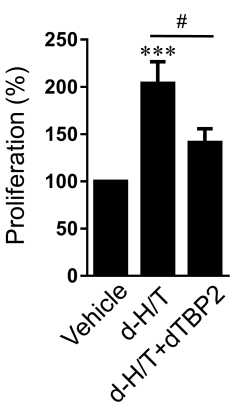

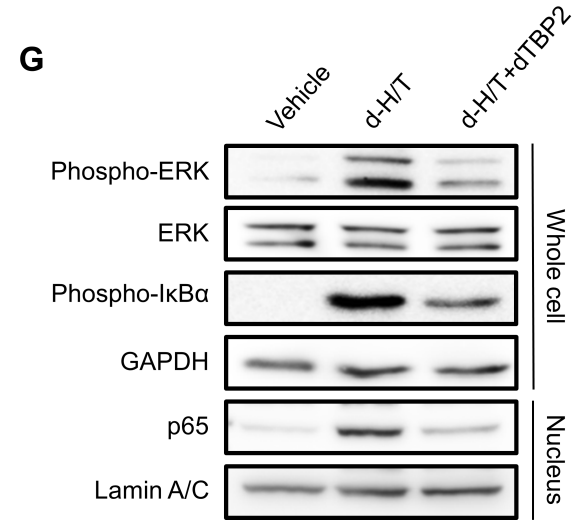

G 
Figure 4
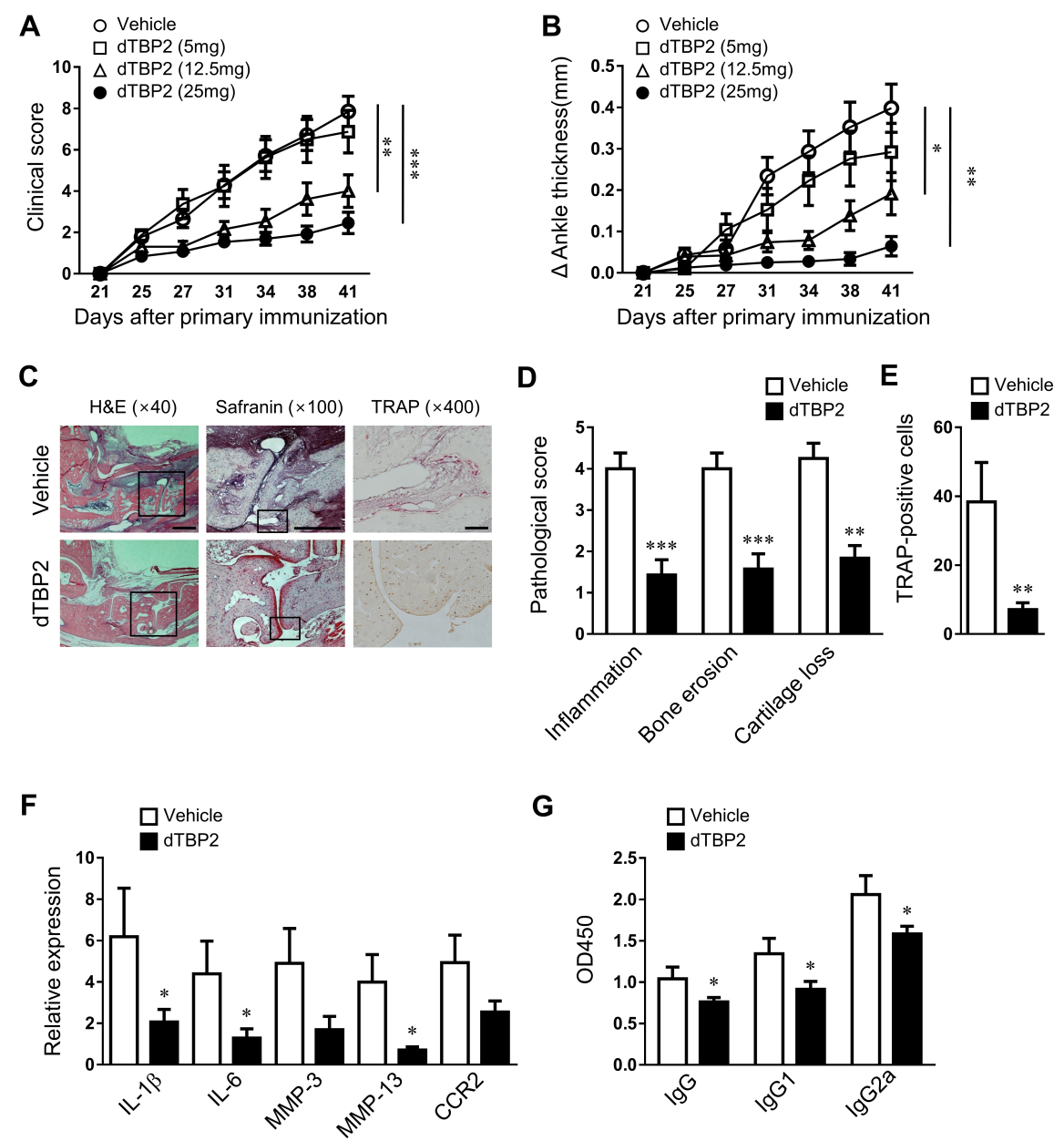
Figure 5
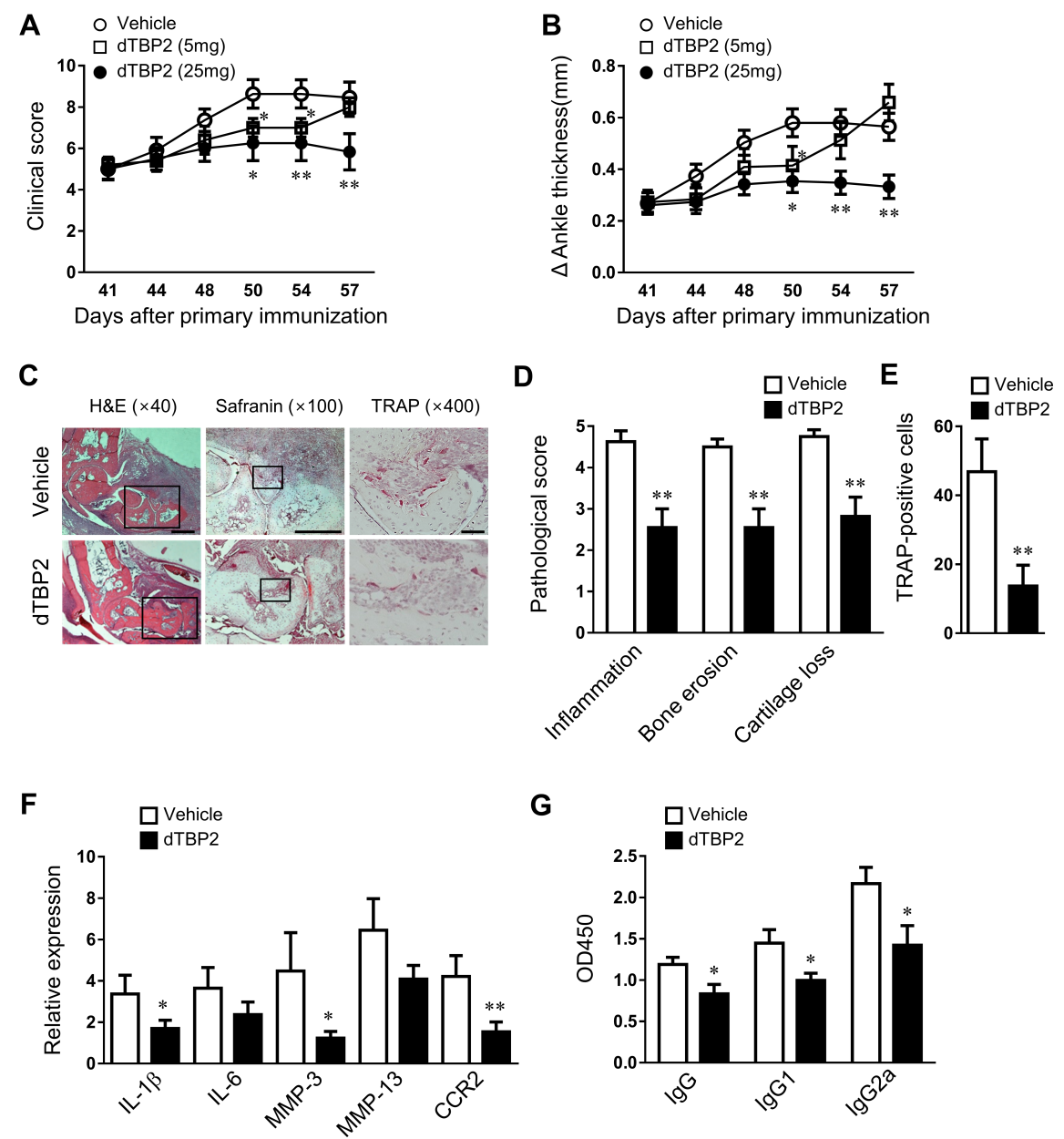
Figure 6
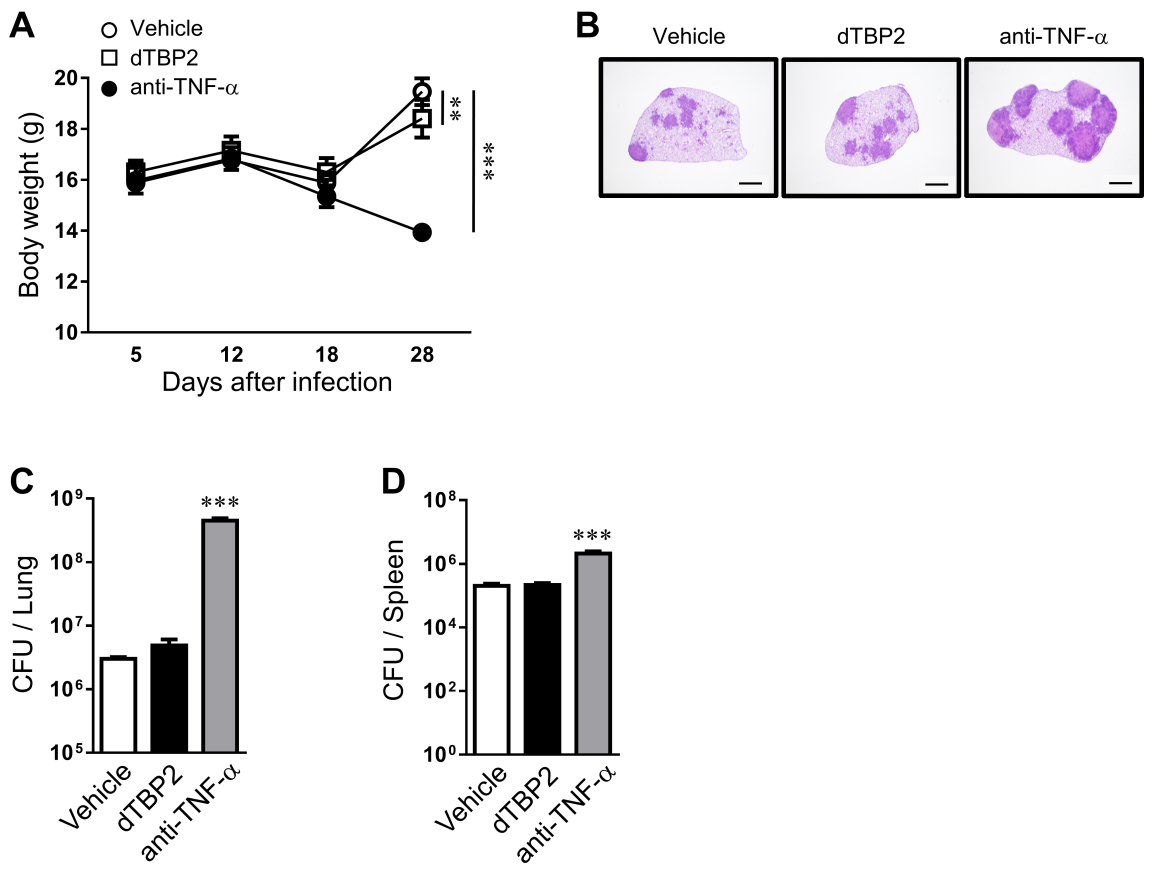